\title{
El índice $h$ de Hirsch: su aplicación a algunos de los científicos españoles más destacados
}

\author{
Por Grupo Scimago
}

Grupo Scimago. "El índice $h$ de Hirsch: su aplicación a algunos de los científicos españoles más destacados”. En: El profesional de la información, 2007, enero-febrero, v. 16, n. 1, pp. 47-49.

http://dx.doi.org/10.3145/epi.2007.ene.05

EN UN NÚMERO ANTERIOR $^{1}$ HABÍAMOS introducido el nuevo y polémico índice $h$, propuesto por Jorge Hirsch ${ }^{2}$, a su vez alabado, criticado y estudiado por los más importantes autores del campo de la bibliometría. Recordemos que la idea que subyace es tan ingeniosa como sencilla. Consiste simplemente en ordenar los trabajos de un autor de forma decreciente en virtud de las citas recibidas por cada trabajo. En el momento en el que el rango (posición en la lista) supera o iguala al valor de la cita, ahí tenemos nuestro índice $h$. Esto significa que el autor tiene $h$ trabajos con al menos $h$ citas $^{3}$.

La mayor parte de los estudios que se han generado en torno a este tema, son en realidad ensayos en los que los expertos reflexionan sobre la validez y efectividad del índice. Sin embargo, en algunos casos se ha comenzado a aplicar el índice para poder analizar sus consecuencias prácticas. Ha sido aplicado en diferentes áreas temáticas, desde la física ${ }^{4}$ hasta la documentación ${ }^{5}$ ${ }^{6}$, como también en distintas áreas geográficas, como Brasil ${ }^{7}$. En este texto lo aplicaremos a una serie de científicos españoles con el fin de hacer consideraciones sobre el comportamiento de este nuevo indicador. En la tabla 1 se listan los nueve científicos escogidos.

Como puede apreciarse, provienen de diferentes disciplinas de las ciencias básicas y aplicadas y algunos figuran en el ISI-highly-cited ${ }^{8}$. En el gráfico 1 vemos la curva de citación de cada uno de ellos.
En el punto de intersección de la curva de citación con la recta que representa el rango, encontramos su índice $h$. A simple vista observamos dos autores claramente separados del resto (Rodés y Barbacid). Además, ambos intercambian sus posiciones, Barbacid arranca con la friolera de 3.811, $1.091 \mathrm{y}$ 1.086 citas en los primeros tres documentos, para finalmente alcanzar un $h=79$. Rodés arranca con sólo 660 citas en su documento más citado, pero, sin embargo, mantiene un nivel sostenido de citación. Su curva cruza la de Barbacid en el documento número 68 , y obtiene un $h=85$. La gráfica sugiere una carrera más consolidada y constante en el segundo caso. Este fenómeno nos da alguna de las pistas de la naturaleza del comportamiento del

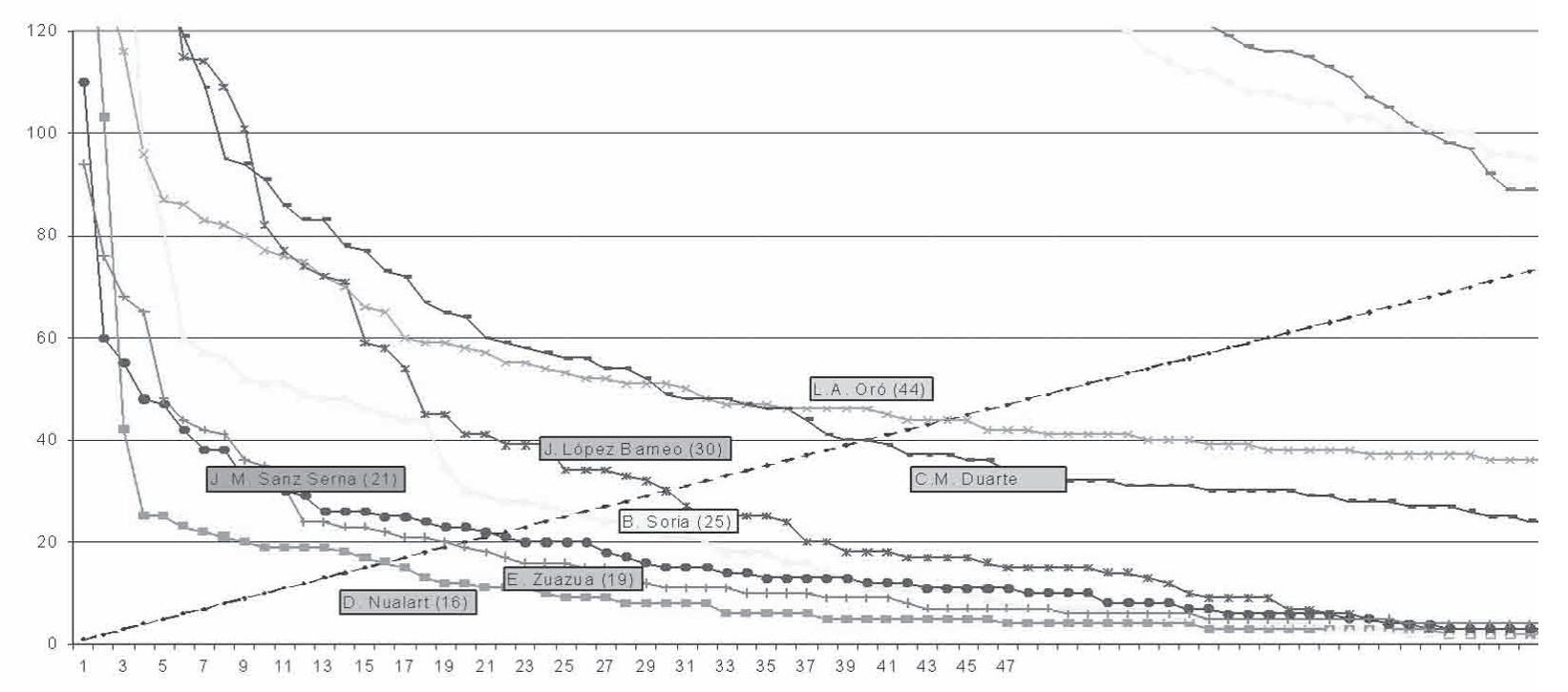

Gráfico 1 


\begin{tabular}{|l|l|l|r|r|r|r|}
\hline \multicolumn{1}{|c|}{ Autor } & \multicolumn{1}{|c|}{ Institución } & \multicolumn{1}{|c|}{ Categoría } & Docs. & Citas & h & Citas/docs \\
\hline Nualart, David & Universidad de Barcelona & Mathematics & 128 & 932 & 16 & 7,28 \\
\hline Soria, Bernat & $\begin{array}{l}\text { Centro Andaluz de Biología } \\
\text { Molecular y Medicina } \\
\text { Regenerativa }\end{array}$ & Cell Biology & 149 & 2123 & 25 & 14,24 \\
\hline Oro, Luis A. & $\begin{array}{l}\text { CSIC-Universidad de } \\
\text { Zaragoza }\end{array}$ & Chemistry & 465 & 9608 & 44 & 20,66 \\
\hline López Barneo, J. & Universidad de Sevilla & $\begin{array}{l}\text { Biochemistry \& } \\
\text { molecular biology }\end{array}$ & 118 & 3121 & 30 & 26,44 \\
\hline Sanz Serna, J. M. & Universidad de Valladolid & Mathematics & 83 & 1333 & 21 & 16,06 \\
\hline Zuazua, Enrique & $\begin{array}{l}\text { Universidad Autónoma de } \\
\text { Madrid }\end{array}$ & Mathematics & 144 & 1315 & 18 & 9,13 \\
\hline Duarte, Carlos M. & CSIC-Univ. IIles Balears & $\begin{array}{l}\text { Plant \& animal } \\
\text { science }\end{array}$ & 255 & 5643 & 40 & 22,12 \\
\hline Barbacid, Mariano & $\begin{array}{l}\text { Centro Nacional de } \\
\text { Investigaciones Oncológicas }\end{array}$ & $\begin{array}{l}\text { Molecular biology } \\
\text { \& genetics }\end{array}$ & 219 & 29022 & 78 & 132,52 \\
\hline Rodes Teixidor, J. & Universidad de Barcelona & Clinical medicine & 1050 & 26096 & 85 & 24,85 \\
\hline
\end{tabular}

$$
\text { Tabla } 7
$$

indicador que habíamos sugerido en el trabajo anterior.

A continuación tenemos a Oro Duarte, cuyas curvas también se interceptan y cambian posiciones. Duarte acumula más citas que Oro en 10 s primeros trabajos, perd mantiene un nivel más parejo número mayor de ellos. De est ma el $h$ es mayor en Oro (44) Duarte (40). Este fenómeno se \$ugiere, pero no llega a cumplirse, en Register for freera at hópez Barneo. Sanz Serna y Zuazua. Finalmente, encontramos a Nualart con el valor de $h$ más bajo. Resulta interesante el escalón de citas que presenta Soria cerca del documento 19. Como esta parte de la curva no ha interceptado la recta del rango, no afecta aún al valor de $h$. Sin embargo, es posible que dentro de poco tiempo, cuando esta zona cruce la recta del rango, el valor de $h$ crecerá rápidamente en función del tamaño del escalón. Por otra parte, este escalón sugiere la existencia de distintas líneas de trabajo con resultados en términos de visibilidad claramente diferentes.

Como hemos indicado, el índice $h$ está en función del esfuerzo y visibilidad de cada uno de los autores, sin embargo, no cabe ninguna duda que la posibilidad de alcanzar un cierto umbral varía en función de las diferentes disciplinas. Los autores que logran valores mayores están dentro de campos muy específicos de la medicina (oncología y hepatología). En el segmento intermedio aparecen otros

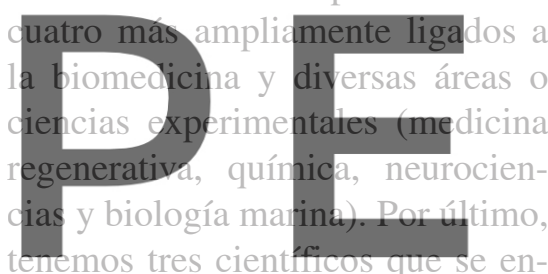

cuentran vinculados más a matemáscipedio

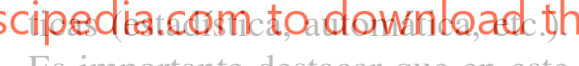
Es importante destacar que en este último campo romper el techo del $h$ es mucho más difícil, tanto por las pautas de citación de la disciplina como por el volumen absoluto de la investigación.

\section{"Los autores que logran valores mayores de $h$ tra- bajan en campos muy es- pecíficos de la medicina"}

Para finalizar hemos calculado, para el caso de estos científicos, la correlación del índice $h$ con el de otros indicadores clásicos. En primer lugar lo hacemos con la cantidad de documentos publicados por cada autor. El valor de $\mathrm{R}^{2}=0,248$, es lo suficientemente bajo como para afirmar que no hay ninguna relación entre ambos indicadores. Esto mismo ocurre cuando correlacionamos citas y documentos, ya que arrojan un $\mathrm{R}^{2}=0,136$, un valor equiparable al anterior y que nos

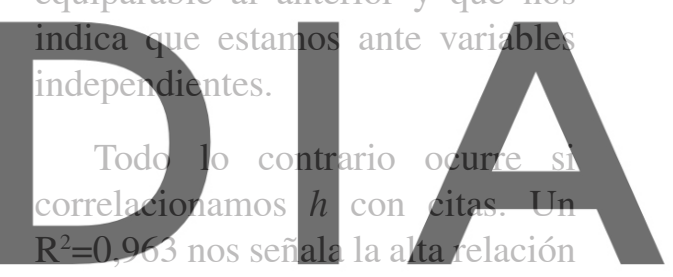

entre ambas variables. En un estu-

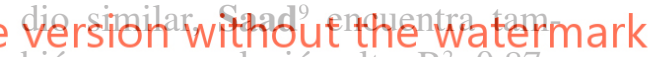
bién una correlación alta: $\mathrm{R}^{2}=0,87$.

Si en cambio comparamos $h$ con citación media (citas/documentos), vemos que es mucho más bajo: $\mathrm{R}^{2}=0,422$. No es lo suficientemente alto, por lo que debemos mantener que las variables son independientes.

Es esta última la correlación más significativa. Como es sabido, la citación media por autor no representa bien la visibilidad internacional media de sus trabajos, ya que la distribución de citas por trabajo no es estadísticamente normal. El factor $h$ aspira a constituirse, sin embargo, en el indicador que exprese mejor el nivel medio de visibilidad internacional de todos los trabajos de un autor, por lo que su creador lo ha llamado "indicador de carrera". 
En índice $h$ de Hirsch: su aplicación a algunos de los científicos más destacados

\section{Notas}

1. Grupo Scimago. "El índice h de Hirsch: aportaciones a un debate". En: El profesional de la información, 2006, julio-agosto, v. 15, n. 4, pp. 304-306.

2. http://physics.ucsd.edu/ jorge/jh.html

3. Hirsch, Jorge. "An index to quantify an individual's scientific research output". En: ArXiv: physics/0508025, 2005. http://arxiv.org/abs/physics/0508025

4. Ball, Philip. "Index aims for fair ranking of scientists". En: Nature, 2005, n. 436, p. 900.
5. Rousseau, Ronald. A case study: evolution of Jasis' Hirsch index, 2006.

http://eprints.rclis.org/archive/00005430/

6. Cronin, Blaise; Meho, Lokman. "Using the h-index to rank influential information scientists". En: Journal of the American Society for Information Science and Technology, 2006, v. 57, n. 9, pp. 1.275-1.278.

7. Batista, P. D.; Campiteli, M. G.; Kinouchi, O.; Martinez, A. S. "Is it possible to compare researchers with different scientific interests?". En: ArXiv: physics/0509048, 2005.

http://arxiv.org/abs/physics/0509048
8. http://isihighlycited.com/

9. Saad, Gad. "Exploring the h-index at the author and journal levels using bibliometric data of productive consumer scholars and business-related journals respectively". En: Scientometrics, 2006, v. 69 , n. 1 , pp. 117-120.

Grupo Scimago (Imago scientiae o visualización de la ciencia). scimago@ugr.es http://www.atlasofscience.net 